\title{
The Influence of LED Headlamp Dissipation System Based on Thermoelectric refrigeration
}

\author{
Sun Rui ${ }^{1, a}$, Xu Xiang-Yang ${ }^{2, b}$ \\ ${ }^{1}$ Beijing Key Laboratory for High-efficient Power Transmission and System Control of New \\ Energy Resource Vehicle, School of Transportation Science and Engineering, Beihang University, \\ 37 Xueyuan Road, Haidian District, Beijing 100191, China \\ ${ }^{2}$ Beijing Key Laboratory for High-efficient Power Transmission and System Control of New \\ Energy Resource Vehicle, School of Transportation Science and Engineering, Beihang University, \\ 37 Xueyuan Road, Haidian District, Beijing 100191, China \\ arichardwilliam@163.com, ${ }^{\mathrm{b}} \mathrm{xxy} @$ buaa.edu.cn
}

Key words: LED; automotive headlamp; heat dissipation; thermoelectric refrigeration.

Abstract. The heat produced in working process of LED headlamp will decrease the LED chips' luminous efficiency and other problems if it is not dispersed in time. This article take thermoelectric refrigeration system as the research object, focus on the relationship between the influencing factors such as LED junction temperature, radiator thermal resistance, temperature difference and refrigeration coefficient, and draw a conclusion at the end.

\section{Introduction}

With the continues improvement of the brightness, the application field of LED are becoming more and more wide. However, a substantial increase in brightness, makes the heat dissipation become the biggest obstacle for commercialization. If the heat can not be dissipated timely and effectively, it will lead to over-high temperature for devices, and a series of evil consequences, such as accelerated aging of packaging materials, accelerated luminous decay which will shortens their lifespan. Meanwhile, a large amount of heat can not be dissipated, the thermal stress will cause damage to the LED chips. Common measures for dissipating heat is to install the LED chips on the substrate directly, which has high thermal conductivity, enhancing the heat dissipation through convection, heat radiation and heat conduction.

In general, according to the different modes of the airflow around the radiator, the heat dissipation modes of LED automotive headlamps can be divided into two categories: passive and active[1]. The passive radiator is a kind of radiator whose main heat dissipation is heat convection. Although it has simple and good stability, the effect of heat dissipation is relatively poor due to low coefficient of natural convective heat transferring.

Active heat dissipating schemes usually include air heat dissipating, heat pipe heat dissipating, semiconductor refrigeration heat dissipating, liquid-dissipation heat dissipating, etc. Air heat dissipating is usually installed with variable speed fan on the back end of the radiator, changing the surrounding airflow, enhancing the heat convection. It has a good effect for heat dissipating in low temperature, but increases the energy consumption and reduces the reliability of the system, so the lifespan and stability of the fan become the lighting system's shortcoming. Heat pipe heat dissipating uses the phase transition process of a closed vacuum tube medium to realize heat transferring. It has a better performance for heat transferring and good isothermal capacity. At the same time, it also has viscous and capillary limits, and limitations of large space occupying. Liquid-dissipation heat dissipating uses the forced circulation for dissipation liquid under the external driving force, to achieve heat convection between the radiator and the dissipation liquid. With high requirements for packaging, its stability is not high, and it must be promoted by pumps to enhance heat dissipating. With limited technology level in domestic liquid-dissipation aspects, if a liquid-circulation refrigeration device is installed on the high-power LED, there will be serious problems in the reliability of the device. It hasn't been approved for research \& development so far. The 
thermoelectric refrigeration heat dissipating, i.e. semiconductor refrigeration heat dissipating, is a thermoelectric refrigeration effect by using semiconductor chips, to maximize the temperature difference between the heat source and radiator, so as to increase the heat conduction. At the same time, thermoelectric refrigeration also applies the Seebeck effect, Peltier effect, Fourier effect, Joule effect and Thomson effect. Thermoelectric refrigerators have the advantages of solid stabilizing, energy saving, small volume, and they are easy to integrate with LED. Therefore, the most promising way to solve the problem for high-power LED's headlamps in heating dissipating is the thermoelectric refrigeration.

\section{The thermal performance and influencing factors of thermoelectric dissipation}

The Thermoelectric Refrigeration Principle. Thermoelectric dissipation heat also known as semiconductor refrigeration, which means achieving the dissipation goals by using thermoelectric dissipation effect(Peltier effect)of the semiconductor chip, in order to maximize the temperature difference between the heat source and heat sink as much as possible, therefore increase the heat conductivity. Thermoelectric refrigerator is a new type of refrigeration equipment based on thermoelectric dissipation effect, because of its compact structure, low noise, and simple control and other advantages, this dissipation method has been widely used in home air conditioners, refrigerators, medical equipment, infrared detection and aerospace technology and etc..

Cold End (connect with LED substrate)

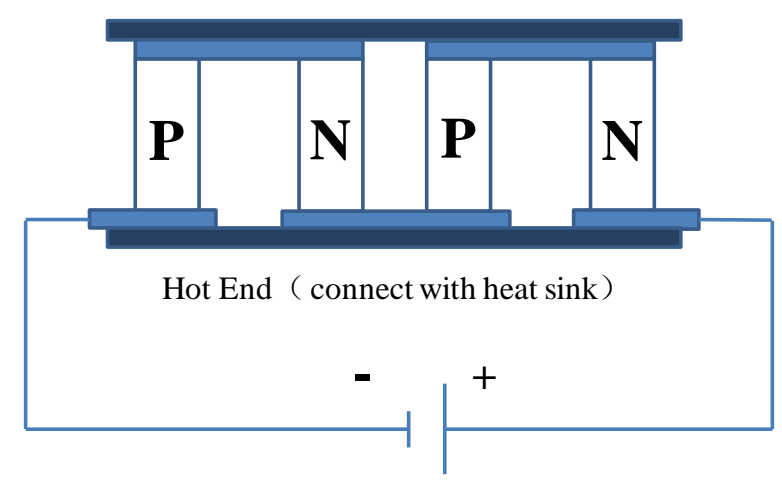

Fig. 1 Operating principle of thermoelectric cooling

Fig. 1 is the operating principle of thermoelectric dissipation system, in which the core part is the semiconductor chip that usually consists of multiple pairs of thermocouples[2]. Circuit consists of drive power, P-type semiconductor, N-type semiconductor, copper and metal plate. P, N-type semiconductor are mutually crosswise arranged, and interconnected by a metal plate, meanwhile, the power source and the semiconductor are connected by the copper, and the entire circuit is basing on Peltier effect. The current direction of the cold end is from $\mathrm{N}$ to $\mathrm{P}$, and connects with LED substrate; And the current direction of the hot end is from $\mathrm{P}$ to $\mathrm{N}$, and connects with heat sink. Series connection of hot and cold end called a thermocouple, so that many thermocouples connected in series constituting a thermopile as shown above[2]-[3]. The heat flow of the hot end which is connected to a heat sink is greater than that of the cold end connected with LED substrate, that could explain why the thermopile can be used for dissipation. When the circuit is turned on, the temperature of the cold end connected with the LED board drops and absorbs heat from the LED substrate at the same time; Similarly, the temperature of the hot end connected with the heat sink rises and releases heat absorbing from the cold end to the environment. A typical thermoelectric dissipation model[4] is shown in Fig. 2: 


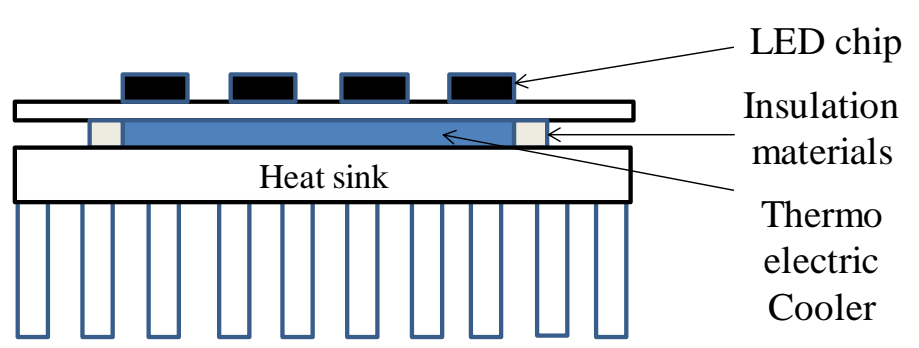

Fig. 2 Typical thermoelectric cooling model

Thermal Simulation. Basing on the principles of thermodynamics and the Peltier effect, we can derive the basic Eq. of the thermoelectric dissipation principle as following[5] Eq. 1 4:

$$
\begin{gathered}
Q_{C}=S_{C} \mathrm{I} T_{c}-\frac{1}{2} I^{2} R_{m}-K_{M} \Delta T \\
P_{D}=S_{C} I \Delta T+I^{2} R_{m} \\
Q_{H}=Q_{C}+P_{D}=S_{C} I T_{H}+\frac{1}{2} I^{2} R_{m}-K_{M} \Delta T \\
\in=\frac{Q_{C}}{P_{D}}=\frac{S_{C} I T_{C}-\frac{1}{2} I^{2} R_{m}-K_{M} \Delta T}{S_{C} I \Delta T+I^{2} R_{m}}
\end{gathered}
$$

In the Eq. 1 Eq. 4, $\mathrm{Q}_{\mathrm{C}}$ represents the dissipation capacity of the cold end of thermoelectric refrigerator, $S_{C}$ represents the Seebeck coefficient, I represents current, $R_{M}$ is dependent resistor, $T_{C}$ represent the cold end temperature of thermoelectric refrigerator, $K_{M}$ represents thermal conductivity, $\Delta T$ represents the temperature difference between the cold end and the hot end, $\mathrm{P}_{\mathrm{D}}$ is the dissipation power of the dissipation system, $\mathrm{Q}_{\mathrm{H}}$ represents the calorific value of the hot end, $\mathrm{T}_{\mathrm{H}}$ is the hot end temperature of thermoelectric refrigerator, and $\varepsilon$ represents the dissipation factor. From the Fig. 2 we can see, the junction temperature of LED chip can be expressed as:

$$
T_{J}=T_{H}-\Delta T+P_{D} R_{J B}
$$

In the Eq. $5, \mathrm{~T}_{\mathrm{B}}$ represents substrate temperature, $\mathrm{P}_{\mathrm{D}}$ represents the dissipation power of the LED elements, $R_{J B}$ represents the thermal resistance between the LED PN junction and the substrate, ignoring the thermal resistance between the substrate and the thermoelectric refrigerator, as well as the thermal resistance between the thermoelectric refrigerator and the heat sink. Since $T_{B}=T_{C}=$ $\mathrm{T}_{\mathrm{H}}-\Delta \mathrm{T}$, substitute it into the above Eq. 5, we can get:

$$
\begin{gathered}
T_{J}=T_{H}-\Delta T+P_{D} R_{J B} \\
T_{H}=T_{A}+Q_{H} R_{J A}
\end{gathered}
$$

In the Eq. 7, $\mathrm{R}_{\mathrm{JA}}$ represents the thermal resistance between the hot end of thermoelectric refrigerator and the environment, that is, the thermal resistance of the heat sink, $\mathrm{T}_{\mathrm{A}}$ represents the ambient temperature. substitute it into the above Eq. 6 , we can get:

$$
T_{J}=T_{A}+Q_{H} R_{J A}-\Delta T+P_{D} R_{J B}
$$

The simultaneous solution for Eq. 1 and Eq. 3 is applied in order to get $\mathrm{Q}_{\mathrm{H}}$ expression as follows, and take positive results:

$$
Q_{H}=\frac{S_{C}^{2}\left(2 T_{H}^{2}-3 \Delta T T_{H}+\Delta T^{2}\right)-\left(2 S_{C} T_{H}-S_{C} \Delta T\right) \sqrt{S_{C}^{2}\left(T_{H}-\Delta T\right)^{2}-2 R_{M}\left(Q_{C}+K_{M} \Delta T\right)}}{R_{M}}-Q_{C}-2 K_{M} \Delta T
$$

Substitute the results into the above Eq. 7:

$$
R_{J A}=\frac{R_{M}\left(T_{H}-T_{A}\right)}{S_{C}^{2}\left(2 T_{H}^{2}-3 \Delta T T_{H}+\Delta T^{2}\right)-\left(2 S_{C} T_{H}-S_{C} \Delta T\right) \sqrt{S_{C}^{2}\left(T_{H}-\Delta T\right)^{2}-2 R_{M}\left(Q_{C}+K_{M} \Delta T\right)}-R_{M}\left(Q_{C}+2 K_{M} \Delta T\right)}
$$

As can be seen from the Eq. 9 , when temperature difference $(\Delta \mathrm{T})$ of the thermoelectric refrigerator between hot and cold ends remains unchanged, there is a function relationship between LED chip junction temperature $\left(T_{J}\right)$ and the thermal resistance of the heat $\operatorname{sink}\left(R_{J A}\right)$; Similarly, from Eq. 4, we 
can obviously draw a conclusion that, when the LED chip junction temperature $\left(\mathrm{T}_{\mathrm{J}}\right)$ and the temperature of the cold end $\left(\mathrm{T}_{\mathrm{C}}\right)$ remain constant, there is also a function relationship between dissipation coefficient $(\varepsilon)$ and the temperature difference $(\Delta \mathrm{T})$ between the hot and cold ends.

If the thermoelectric refrigerator in the typical thermoelectric dissipation model is removed, then the expressions of the junction temperature for LED chip are as follows:

$$
T_{J}=T_{A}+P_{D} R_{\mathrm{BA}}+P_{D} R_{\mathrm{JB}}
$$

As can be seen from the Eq. 10, there exists a functional relationship between $T_{J}$ and $R_{B A}$.

Take typical German Schefenacker lighting module designed as example[6], explore the thermal performance of thermoelectric dissipation, the relevant parameters are as follows: $\mathrm{S}_{\mathrm{C}}$ is $0.05253 \mathrm{~V} / \mathrm{K}$, the resistance $\mathrm{R}_{\mathrm{M}}$ is $0.4835 \Omega$, the thermal conductivity is $2.8074 \mathrm{~W} / \mathrm{K}$, power dissipation of LED is $75 \mathrm{~W}$, the thermal resistance choose $15 \mathrm{~K} / \mathrm{W}$, then $\mathrm{R}$ is about $0.2 \mathrm{~K} / \mathrm{W}$, the ambient temperature is $20^{\circ} \mathrm{C}$, according to the derived Eq. 1 to Eq. 10 above, calculated the changing data come from the thermal resistance of the heat sink, junction temperature of the LED chip and dissipation coefficient of the thermoelectric refrigerator along with temperature difference between hot and cold end. Data is plotted in Table 1:

Table 1 Results of LED Thermal simulation

\begin{tabular}{c|c|c|c|c|c|c|c|c}
\hline & \multicolumn{2}{|c|}{$\Delta \mathrm{T}=0\left[{ }^{\circ} \mathrm{C}\right]$} & \multicolumn{2}{c|}{$\Delta \mathrm{T}=10\left[{ }^{\circ} \mathrm{C}\right]$} & \multicolumn{2}{c|}{$\Delta \mathrm{T}=20\left[{ }^{\circ} \mathrm{C}\right]$} & \multicolumn{2}{c}{$\Delta \mathrm{T}=30\left[{ }^{\circ} \mathrm{C}\right]$} \\
\cline { 2 - 9 } & $\mathrm{T}_{\mathrm{J}}\left[{ }^{\circ} \mathrm{C}\right]$ & $\varepsilon$ & $\mathrm{T}_{\mathrm{J}}\left[{ }^{\circ} \mathrm{C}\right]$ & $\varepsilon$ & $\mathrm{T}_{\mathrm{J}}\left[{ }^{\circ} \mathrm{C}\right]$ & $\varepsilon$ & $\mathrm{T}_{\mathrm{J}}\left[{ }^{\circ} \mathrm{C}\right]$ & $\varepsilon$ \\
\hline $\mathrm{R}_{\mathrm{JA}} / \Omega=0.1$ & 42.5 & $\backslash$ & 35.8 & 2.16 & 29.1 & 0.99 & 21.3 & 0.58 \\
\hline $\mathrm{R}_{\mathrm{JA}} / \Omega=0.2$ & 50 & $\backslash$ & 46.3 & 2.32 & 42 & 1.19 & 41.7 & 0.69 \\
\hline $\mathrm{R}_{\mathrm{JA}} / \Omega=0.3$ & 57.5 & $\backslash$ & 56 & 2.50 & 54 & 1.32 & 53.8 & 0.78 \\
\hline
\end{tabular}

As can be seen from the data in the table, when $\mathrm{R}_{\mathrm{JA}}$ remain constant, LED chip junction temperature $T_{\mathrm{J}}$, dissipation coefficient $\varepsilon$ of the thermoelectric refrigerator and the temperature difference between hot and cold end $\Delta \mathrm{T}$ showed a negative correlation, that is, $\mathrm{T}_{\mathrm{J}}$ and $\varepsilon$ diminishes as the value of $\Delta \mathrm{T}$ rises; when $\Delta \mathrm{T}$ remains unchanged, junction temperature $\mathrm{T}_{\mathrm{J}}$, dissipation coefficient $\varepsilon$ and thermal resistance $\mathrm{R}_{\mathrm{JA}}$ showed a positive correlation, namely $\mathrm{T}_{\mathrm{J}}$ and $\varepsilon$ increase with the value of $\mathrm{R}_{\mathrm{JA}}$ rises; If the thermoelectric refrigerator in the typical thermoelectric dissipation model is removed, according to the Eq. 10 above, it can be seen, $\mathrm{T}_{\mathrm{J}}$ reaches its maximum. Therefore, the dissipation effect of the thermoelectric refrigerator can be proved, and for LED headlamp, the dissipation effect of the thermoelectric refrigerator is relatively prominent. In order to analyze the relationship between the thermal resistance of heat sink, the junction temperature of LED chip and dissipation coefficients more intuitively and their changes along with the temperature difference of the hot and cold ends $\Delta \mathrm{T}$, we plot into a figure by using acquired data.

Influencing Factors. First, draw a relation curve of $\mathrm{R}_{\mathrm{JA}}$ and $\mathrm{T}_{\mathrm{J}}$, when $\Delta \mathrm{T}$ is $0^{\circ} \mathrm{C}, 10^{\circ} \mathrm{C}, 20^{\circ} \mathrm{C}$ and $30^{\circ} \mathrm{C}$, relation between $\mathrm{R}_{\mathrm{JA}}$ and $\mathrm{T}_{\mathrm{J}}$ shown in Fig. 3: 


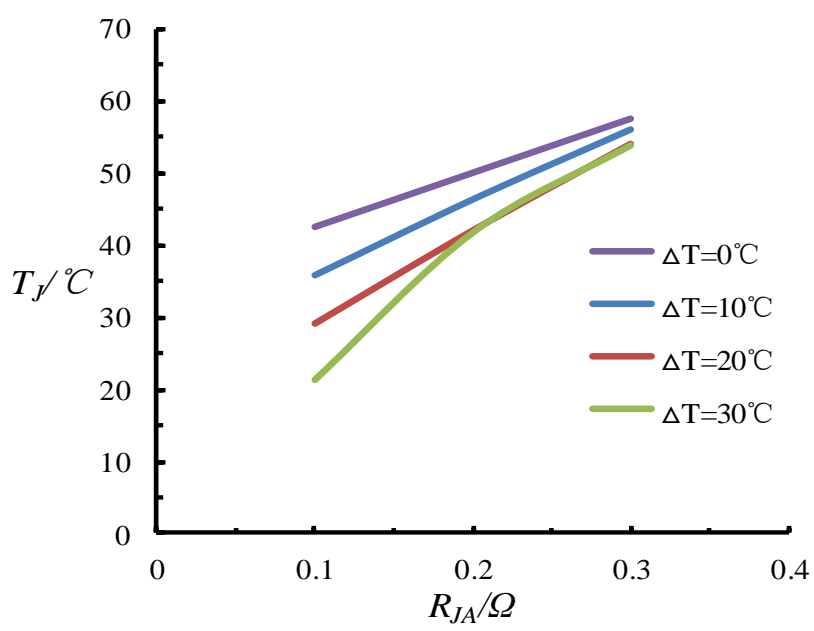

Fig. 3 The relation curve of junction temperature and thermal resistance

From the Fig. 3 we can see, if the thermoelectric refrigerator in the typical thermoelectric dissipation model is removed, which uses passive dissipation system, if $\Delta \mathrm{T}$ remains unchanged, the junction temperature of LED chip $T_{J}$ and the thermal resistance of heat sink $R_{J A}$ showed a positive correlation. that $T_{J}$ increases with the value of $R_{J A}$ rises, the slope of the curve represents the power of the LED chip. For the LED headlamp dissipation system using thermoelectric refrigerator, $\mathrm{T}_{\mathrm{J}}$ and $\mathrm{R}_{\mathrm{JA}}$ also showed a positive correlation. At the same time, we can find slope of the curve become bigger when $\Delta \mathrm{T}$ increases, so does the power and temperature of LED chip. The Fig. 3 shows that the temperature curve of passive dissipation system and the temperature curve of thermoelectric refrigerator dissipation system have an intersection, namely the turning point of thermal resistance between the hot end of the thermoelectric refrigerator and the environment, represented by $\mathrm{R}_{\text {JAmax }}$. If you want to reduce junction temperature by using thermoelectric refrigerator, the value of the thermal resistance between the hot end of the thermoelectric refrigerator and the environment should be less than the value of the turning point, otherwise, dissipation effect of the refrigerator will be lost. At the same time, we can see the location of $\mathrm{R}_{\mathrm{JAmax}}$ varies with the $\Delta \mathrm{T}$, presents the negative relationship overall, which is, $R_{J A m a x}$ diminishes as the value of $\Delta T$ rises. $R_{J A m a x}$ is an vital parameter in the design of the dissipation system and should be given adequate attention.

Then, draw a relation curve of $\Delta \mathrm{T}$ and $\varepsilon$, when $\mathrm{T}_{\mathrm{J}}$ is $30^{\circ} \mathrm{C}, 40^{\circ} \mathrm{C}$ and $50^{\circ} \mathrm{C}$, relation between $\Delta \mathrm{T}$ and $\varepsilon$ shown in Fig. 4:

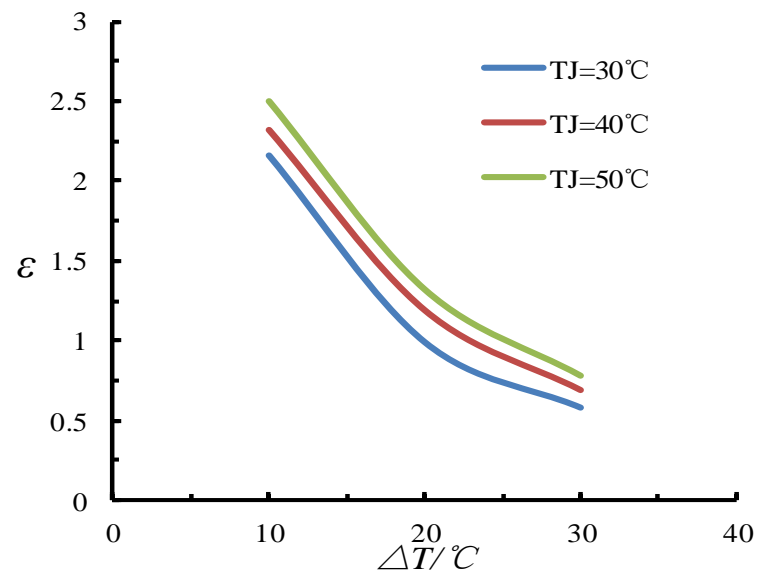

Fig. 4 The relation curve of junction temperature and thermal resistance

From the Fig. 4, it is clear that when $T_{J}$ remains unchanged, dissipation coefficient $\varepsilon$ and the temperature difference between hot and cold end $\Delta \mathrm{T}$ has a negative correlation, which is, $\varepsilon$ diminishes as the value of $\Delta \mathrm{T}$ rises, but not decreases linearly, which is, the bigger the value of $\Delta \mathrm{T}$ is, the less the extent of $\varepsilon$ decrease is.

The reason for this phenomenon is, $\Delta \mathrm{T}$ increase, current intensity and the power of LED chips rise at the same time, and the heat generated by the system will also increase, so $\varepsilon$ becomes smaller. When 
the value of $\Delta \mathrm{T}$ is small, the dissipation power of the system is much larger than the dissipation power, according to Eq. 4, when the value of the dissipation power is large, dissipation coefficient is also large, speed of the reducing is faster, but with the increasing of temperature difference between the hot and cold ends, the dissipation power will continue to increase, the rate of increase of the dissipation power is much larger than that of the dissipation power, so is why the refrigeration coefficient decreases more slowly. In the design of the dissipation system, we need to control the thermal resistance of the heat sink, so that the temperature difference between hot and cold end could be controlled in a smaller value, in order to make refrigeration coefficient of dissipation systems relatively large, by doing this, dissipation efficiency of the system can be improved as well.

In summary, in the thermoelectric refrigeration system of the LED headlamp, under the definition of the value of the thermal resistance between the hot end of the thermoelectric refrigerator and the environment remaining constant, the greater the temperature difference between hot and cold end is, the lower the junction temperature of LED chip is, but there is a downside in this way, which is the dissipation coefficient would be reduced at the same time, the dissipation efficiency is not ideal; If the range of the temperature difference between hot and cold ends is small, although refrigeration coefficient would be more ideal, the junction temperature of LED chip is still relatively high, relatively speaking, and the dissipation effect is equally unsatisfactory.

Therefore, the temperature difference between the hot and cold ends should be neither too big nor too small, should be controlled within a certain range, so it could not only ensure the thermoelectric refrigeration coefficient is ideal, but also control the junction temperature of LED chip in a relatively low range, to achieve a relatively stable equilibrium. Under the conditions of sufficient space, using a multi-stage thermoelectric cooler could be considered, take the small cumulative temperature difference for many times, ultimately control the refrigeration coefficient and junction temperature of LED chip within the desired range.

\section{Conclusion}

In this paper, through the study of junction temperature, thermal resistance, temperature difference between hot and cold sides and refrigeration coefficient, we found that the LED system with thermoelectric refrigeration has a maximum $-\mathrm{R}_{\mathrm{JAmax}}$ for radiator's thermal resistance, and the thermoelectric refrigeration system can reduce the LED junction temperature only less than this value; there is a balance between refrigeration coefficient and the LED junction temperature, and the effective range of temperature difference $\Delta \mathrm{T}$ should be considered in practice. The study also found that using multilevel refrigeration can obtain a better effect for heat dissipating. In short, the use of semiconductor refrigeration mode to solve the problem of heat dissipation for high-power LED is indeed feasible, and its effect is also very obvious. At the same time, it can be adjusted according to the actual situation, to improve the accuracy by the use of closed-loop temperature controlling circuit. What's more, it has a small size, light weight, high reliability, and easy to achieve. Therefore, such heat dissipation mode has a very good development prospect, and it is possible to come into being industrialized in the near future. With the continuous development of semiconductor technology and process, to solve the heat dissipation problems of LED automotive lighting by this approach will have more practical value.

\section{Acknowledgements}

This work was supported by the Fundamental Research Funds for the Central Universities, and was also supported by the Beijing Key Laboratory for High-efficient Power Transmission and System Control of New Energy Resource Vehicle. 


\section{References}

[1] Gu Y, Narendran N. A noncontact method for determining junction temperature of phosphor-converted white LEDs[C]//Optical Science and Technology, SPIE's 48th Annual Meeting. International Society for Optics and Photonics, 2004: 107-114.

[2] Jiin-Yuh Jang, Ying-Chi Tsai, Chan-Wei Wu. A study of 3-D numerical simulation and comparison with experimental results on turbulent flow of venting flue gas using thermoelectric generator modules and plate fin heat sink [J]. Energy, 2013, 53: 270 281.

[3] Y. Sungtaek Ju. Solid-State Refrigeration Based on the Electro caloric Effect for Electronics Cooling[J]. Journal of Electronic Packaging, 2010, 132: 041004-1 041004-6.

[4] Dai Yuan-De, et al. The experimental research of heat pipe radiator semiconductor refrigeration system [J]. Journal of Nanchang University, 2013, 35(1): 54-57.

[5] Zheng Tong-Chang. High power LED module thermal simulation of semiconductor refrigeration[J]. photoelectric device, 2010, 31(2): 210-212.

[6] Qi Chen-Jie. Experimental study about the status of the optimization design of semiconductor refrigerating machinery $[\mathrm{J}]$. Cryogenics, 2007(1): 43-46. 